\title{
How to use your smartphone for outreach
}

\section{Nellist ${ }^{a, *}$}

${ }^{a}$ Institute for Mathematics, Astrophysics and Particle Physics, Radboud University / Nikhef, Nijmegen, Netherlands.

E-mail: clara.nellist@cern.ch

The best camera is the one you have on you.

In this tutorial, I discussed how to use a smart phone to communicate scientific work with a remote audience using the inbuilt camera to produce still images and video content. A description of how this content can then be edited, if needed, and shared on various social media platforms was presented with an alternative option to use a phone to directly live-stream and interact with an audience in real-time.

Also explored in the tutorial was how a communicator can ensure that what they share will be accessible and consider which platforms are better for different forms of content. Finally, how to evaluate the different types of feedback from the platforms and the audience was considered.

*** The European Physical Society Conference on High Energy Physics (EPS-HEP2021), *** *** 26-30 July $2021 * * *$

*** Online conference, jointly organized by Universität Hamburg and the research center DESY ***

\footnotetext{
${ }^{*}$ Speaker
} 


\section{Why?}

It's a good question.

\subsection{Why do science communication?}

Let us start with why someone might want to communicate science. There can be a number of different reasons and they will not all apply to everyone. Below is a non-exhaustive list of why science communication is done.

- Present research results to a wider audience.

- Improve general interest in particle physics.

- Reach potential new colleagues who are interested in pursuing a career in particle physics.

- Present a human side to science and how science is done.

- Encourage governments to support scientific endeavours.

- Gain valuable communication skills.

- Because it's fun and rewarding.

- A reminder that our research is cool!

\subsection{Why use photos and videos in science communication?}

The best equipment is whatever you have on you. Many people now carry smartphones with them all the time and they usually have great cameras and recording capabilities.

It has been shown that using an image or video can help a post on social media to stand out from the crowd, compared to text only [1]. Increased engagement has been shown for posts on twitter with images. Visual content can also provide additional aesthetic or information to compliment what is in the text. Indeed, I have included Figure 1 (and many other photos and images in the tutorial itself) which likely encouraged people to be more engaged with the content of the tutorial. However, it is important to note that a poor or confusing image or video could distract from the content being shared.

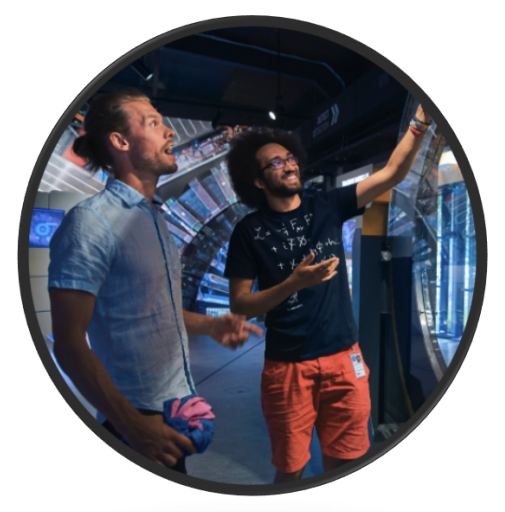

Figure 1: This photo has been included in these proceedings because it will likely help to encourage readers to continue past the first page. (Image: CERN) 


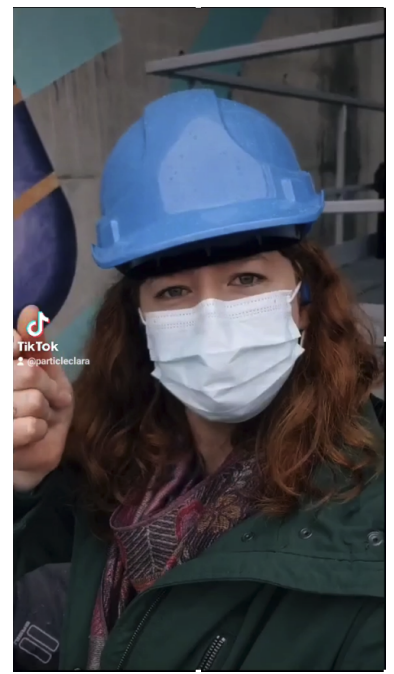

Figure 2: A screenshot from an example video played during the tutorial. The video is a 60 second long compilation of a number of different shots showing a scientist travelling to CERN by bike and heading down to the ATLAS cavern.

More and more people are consuming content on mobile devices and therefore the way that photos and videos are framed need to match how people will watch it. Videos are often filmed in portrait mode, as this is how people will view them on their phones (it is surprisingly difficult to get people to turn their phone around for short videos. They may do it for something longer). Photos should be in a square format, or now also sometimes portrait mode, to fill the space of the screen available.

\subsection{Why am I giving this tutorial?}

I have been working in science communication and outreach for over a decade now. My speciality is social media and content creation. I was the social media manager for the ATLAS experiment at CERN for 5 years and worked with many different social media platforms. I was the first scientist or science communicator from CERN to make dedicated science videos on TikTok.

\section{Content creation}

An example video shown during the tutorial is illustrated in Figure 2 [2]. The video, which was posted on TikTok on the account @ ParticleClara, is a 60 second long compilation of a number of different shots showing a scientist travelling to CERN by bike and heading down to the ATLAS cavern.

If you want to get a little fancier, then you're going to want to download additional applications to help you to edit your content. This can all be done directly on your phone!

All social media apps have some built-in editing capabilities. The best I've found are: Instagram and TikTok. Recommended apps I use (not sponsored :D):

- Capcut: video editing. Can film in my phone's original camera app and then drop the best takes in here.

- OtterAI: creates a transcript of my audio which I then edit for accuracy. 
- Canva: create diagrams and images to explain a concept. This was used to create the image in Figure 3.

- Lightroom: If I want more control to edit my photos.

An example structure for a 30-60 second video is as follows:

1. A hook: Get your audience's attention within 3 seconds

2. The content of the video: pick up to 3 things you want your audience to learn or remember. Too much information and they will be overwhelmed.

3. The round up: summarise what they've learned and consider a call to action.

You can keep your images for use later in presentations or longer articles, but you can also upload it directly online.

Many social media platforms such as TikTok, Instagram in the form of Reels and Stories, and YouTube Shorts are chasing the short video form, as it is very popular right now. You can also stream live events, such as tours, and share behind the scenes directly with followers with your phone.

However, a word of caution: please follow the guidelines of your collaboration, lab, institute, etc, regarding photography and filming rules. If in doubt, check with the relevant outreach or communication team. Also, please wear appropriate safety equipment in all footage.

\section{Accessibility is important}

Since we want to share our work with as many people as possible, this also means making sure that it is accessible to as many people as possible. For visual content this includes, but is certainly not limited to, the following. Adding a description of the image in words, known as alt-text, for visually impaired people. Providing captioning for your videos, which is a transcript of the spoken audio as text overlaid on the video. In addition, a description of the music used can also add layers to the context of the video. This is provided primarily for people who are deaf or hard of hearing, but can also benefit people who are not fluent in the language the video is recorded in. Additionally, many people watch videos when they cannot turn the sound on and this will enable them to watch the video in this situation too.

\section{Evaluation}

It's important to evaluate the content you are putting out to see if it's having the impact that you want it to have.

Some platforms also tell you about your audience. This can be useful to see if you are reaching the target audience you would like to reach. Furthermore, you can compare results against the information for the platform as a whole. For example, TikTok generally has a younger user-base than Twitter, and Instagram has a higher percentage of women users than YouTube.

This can vary depending on your own goals, but often we look at engagement to see if people are looking at your content and liking or commenting. Are the comments positive or negative? 
Negative comments aren't always bad per se. They can be an opportunity to learn and improve. Though, I wouldn't recommend feeding the trolls, so it's also up to you to decide how much you want to interact with people leaving unconstructive negative comments.

In general, it's useful to look at the analytics from the social media platform and keep track to see how your content performs over time.

\section{The Challenge}

During the tutorial, I challenged the attendees to create a video on the topic of their choice. If you are reading these proceedings, why not try it yourself too?

The rules:

- Think of the three main points you want your audience to learn or to remember.

- Catch them in the first 3 seconds!

- Keep it less than 60 seconds long.

- Film in portrait.

- Have fun with it!

If you make one, make sure to let me know!

\section{Bonus}

You don't need a lot of fancy equipment to make good content. But here are a few things that can make things a little easier for you:

- A ring light. This evenly distributes soft light over the presenter and can help the audience to focus on you better.

- A microphone. Sound quality is very important. A word of caution, you should absolutely test this before recording something that is time-critical.

- A tripod. This is great for keeping your phone steady, or placing it away from you so you can film without holding the camera.

\section{References}

[1] P. Ross, Photos get the Most Engagement on Twitter. In: Socialbakers. 21-11-2014. http://www. socialbakers.com/blog/

2306-photos-get-the-most-engagement-on-twitter

[2] https://www.tiktok.com/@particleclara/video/6922959130617466118 


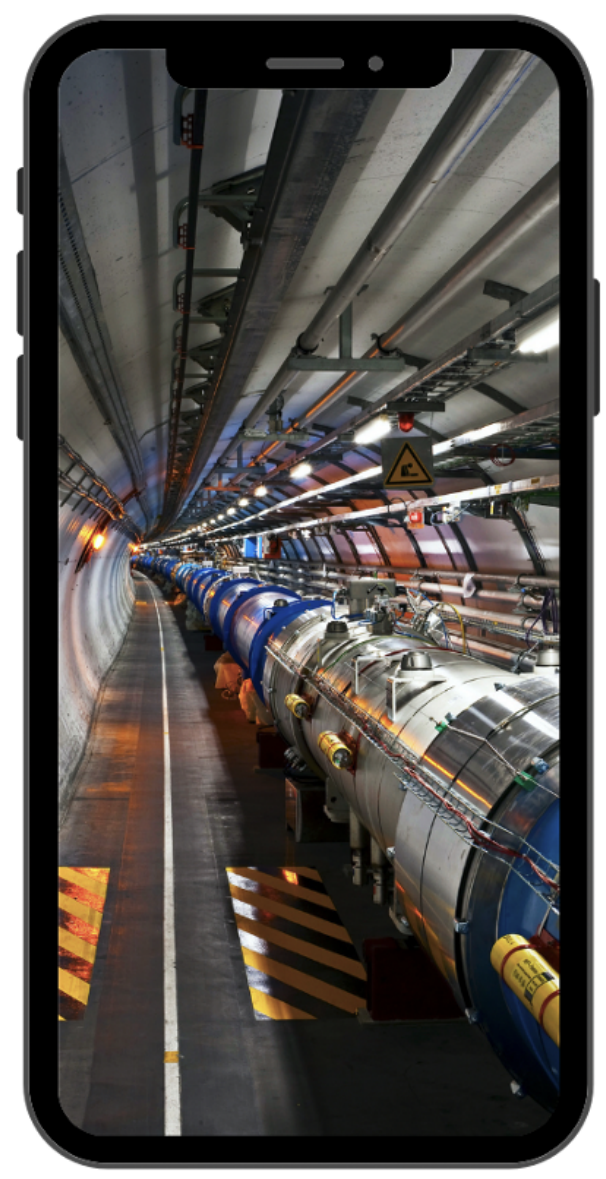

Figure 3: I had some space left so I thought I'd fill it with this nice composite image of the LHC shown on phone as a reward for getting to the end of the proceedings. (Image: CERN, composition: C. Nellist) 